\title{
¿Es ética la utilización de técnicas de simulación en la docencia médica de pregrado? Reflexión bioética
}

\author{
ALBERTO ROJAS O.*y HERNÁN BORJA R.*
}

\section{Is ethical to use simulation techniques in undergraduate medical teaching?}

The use of simulation models in undergraduate medical teaching is now a reality in Chile. This technology provides scenarios of different complexities, and it has a number of advantages for patients' security as well as comfort. The main benefits, limitations and risks of these simulation techniques are pointed out in this article. It is concluded that its use in teaching medical students is not only ethical, but also an indispensable complement in medical education.

Key words: Simulation models, medical education, ethics.

\section{Resumen}

La utilización de modelos de simulación en la docencia de pregrado en medicina ya es una realidad en nuestro país. Pone al alcance del estudiante diversos escenarios de la atención médica, a distintos niveles de complejidad. Tiene muchas ventajas y evita molestias e incomodidades a los pacientes reales. Se proponen sus principales ventajas y riesgos y se concluye que no sólo es ética su utilización, sino que además es un complemento docente indispensable.

Palabras clave: Simulación, educación médica, ética.

La utilización docente en medicina de la técnica de modelos de simulación para enseñar y poner en práctica diversos métodos de abordaje a un paciente idealizado, ha adquirido progresiva importancia. Históricamente, los escenarios de reanimación cardiorrespiratoria o, en obstetricia, la demostración de los distintos momentos del parto con la utilización de muñecos, son dos buenos clásicos ejemplos de la utilidad que han prestado en la educación médica, hace ya bastantes años. $\mathrm{Su}$ aceptación es indiscutible, ya que permiten un buen acercamiento a la realidad de situaciones complejas y dinámicas, las cuales, en circunstancias reales con pacientes de "carne y hueso" se tornan casi imposibles, o al menos de muy escaso acceso a la práctica docente.

Estos métodos hoy gozan de gran aceptación en la docencia de pregrado en medicina y, pese a que en principio estaban reservados para situaciones de emergencia, hoy han cubierto también otros campos, como, por ejemplo, en semiología la auscultación de ruidos cardiopulmonares. Por otra parte, en los años recientes, se ha perfeccionado el método de simulación docente, incluyendo actores para graficar distintos escenarios de la medicina cotidiana, así como en exámenes prácticos a los estudiantes. Una de las principales ventajas de la evaluación con modelos vivos, es que todos los alumnos son sometidos a un examen y examinador similar, evitando sesgos o interpretaciones menos objetivas en los resultados.

No existe una sola razón concreta por la cual estas técnicas han tenido un éxito importante, pero en nuestra opinión ha sido relevante la cultura de la protección del paciente en todas las fases del entrenamiento y de la práctica de la atención sanitaria. Desde este último punto de vista incluso se exige que el adecuado entrenamiento previo al contacto con personas reales, constituye una obligación moral hacia ellos. Por ende, dejar de hacerlo no sería ético. El respeto al mejor interés del paciente no sólo es una cuestión de justicia moral, sino que también nos humaniza como miembros de la sociedad.

* Facultad de Medicina, Universidad Andrés Bello, Sede Viña del Mar, Chile. 
Otra razón que advertimos es la progresiva dificultad de la docencia médica en seres humanos, no sólo desde el punto de vista de la actividad práctica, sino que además por el advenimiento de una serie de procesos que tienen que ver con el consentimiento informado y la privacidad de las personas. Por otra parte, con el aumento de escuelas de medicina, la utilización adecuada de campos clínicos con pacientes reales se hace cada vez más difícil. No es posible ni prudente plantear que un mismo paciente sea entrevistado y evaluado -en ocasiones con preguntas sobre temas íntimos y de suyo sensibles- por varios alumnos en una misma jornada, mucho menos en aspectos que se vinculan con maniobras invasivas.

Sin embargo, en el otro extremo, pensamos que no se puede desconocer -ni mucho menos suspender- la docencia médica en su modalidad clásica, precisamente aprendiendo en y desde el cuerpo y la psiquis del propio enfermo. Es claro que la comunicación de la biografía del paciente en primera persona y la objetivación de sus signos y síntomas no pueden ser superados por modelos, aun usando los de la mejor calidad disponible. Además, cada enfermo es una globalidad, donde su problema personal o dolencia física, está inmerso en un contexto biográfico, social y sobre todo familiar, y ninguno de estos aspectos quedará suficientemente abarcado y comprendido en modelos de simulación.

Por otra parte, dado que el diseño de modelos de simulación ha experimentado un gran avance, hoy es posible disponer de equipos con un alto grado de sofisticación técnica y electrónica avanzada. Su utilización ha ido en constante progreso, siendo especialmente apropiados para el ejercicio de manejo de catástrofes, accidentes de la vía pública o simulación de atención de heridos en forma masiva. El grado de sofisticación alcanzado ha permitido su utilización en cursos, como "ATLS" (soporte vital en trauma avanzado), por ejemplo, con excelentes resultados por el grado de realismo que otorgan esos modelos.

Otra variable en el desarrollo de la metodología de la simulación clínica, como ya hemos dicho, es la inclusión de actores que desempeñan roles precisos, fruto de entrenamiento de gran calidad. Ello ha demostrado ser muy útil en la docencia dirigida, así como en exámenes finales de asignaturas e internados, donde se prestan muy bien para evaluar adecuadamente las habilidades y competencias adquiridas durante el transcurso del currículo. En esto es necesario enfatizar que se requiere de un entrenamiento en simulación clínica previo de excelente nivel, ya que se su- pone que el modelo vivo deberá comportarse tal como se observa en la realidad una persona enferma.

\section{¿Son iguales todos los modelos de simulación y los protocolos para su uso docente?}

Por ahora, cada escuela de medicina posee sus propios sistemas de simulación, en los que pone aquellos énfasis que estima convenientes y que le permiten sus recursos. En consecuencia, no es posible aún percibir al respecto un currículo o "asignaturas" bien definidas, que permita una adecuada comparación entre las escuelas que lo poseen. Una diferencia importante consiste en la calidad, costo y complejidad de los sistemas de simulación que ofrece el mercado actualmente. Se puede establecer una diferencia entre sistemas de baja y alta tecnología. A mayor tecnología y complejidad, para imitar la realidad, el costo de adquirir y operar, así como mantener los equipos se incrementa en forma considerable. En este sentido, existe una excelente revisión de Pelés y Gomar, de la Universidad de Salamanca ${ }^{1}$.

Estamos conscientes que nada puede reemplazar al paciente de "carne y hueso", pero debe reconocerse que la estandarización de ciertas técnicas, como el masaje cardíaco, intubación de la vía aérea, accesos venosos y arteriales, varios tipos de drenajes y punciones, así como la posibilidad cierta de la auscultación cardíaca o pulmonar, pueden realizarse en estos modelos humanos cuantas veces sea necesario, hasta lograr un grado de operación que se estime adecuado, antes de practicar estas técnicas en seres humanos. No es materia de este artículo, pero ya es una realidad la existencia de interesantes modelos de postítulo para prácticas de maniobras quirúrgicas más complejas.

Por lo tanto, este sistema de docencia nos parece que aporta algunas ventajas, que mencionaremos sucintamente.

1. Permite adquirir habilidades en forma estandarizada.

2. Su nivel de acercamiento a la realidad en algunos casos es inmejorable.

3. Permite al equipo docente evaluar los procesos de enseñanza y aprendizaje.

4. Facilita la toma de exámenes con un grado de complejidad similar para todos los estudiantes.

5. El sistema es susceptible de revisión en forma permanente, motivando un excelente proceso de autocrítica.

6. Dado el carácter de laboratorio que representan, pueden ser repetidos tantas veces como sea necesario. 
7. Pueden reproducir y construir distintos escenarios de alta complejidad, como los percibidos en la vida real.

8. Finalmente y muy importante de destacar, es que evita molestias y malestar a personas enfermas reales, a quienes no se provocará incomodidades ni eventuales daños, en el proceso docente.

Con todo, el propósito de esta reflexión es lograr una aproximación a si es ética la utilización de este tipo de medicina de modelos, en la formación de un estudiante de medicina en pregrado. Esto ha sido abordado en la literatura por varios autores y existe acuerdo en que la enseñanza basada en simulación es consistente con las teorías éticas (tópico revisado recientemente por Gul Pinar y Sibel Peksoy) ${ }^{2}$, y la relación entre este quehacer y los paradigmas de beneficencia-no maleficencia y autonomía, de la bioética principialista, incluyendo a la justicia distributiva. En todos estos ámbitos la mirada es positiva. Sin embargo, es necesario destacar que, más allá de sus beneficios, parece importante revisar posibles inconvenientes y limitaciones que advertimos en esta herramienta docente.

Un primer riesgo es el derivado de los mecanicismos. Antes de ejecutar un determinado procedimiento y aplicar una bien estudiada cadena de acciones, es necesario comprender que se está actuando frente a la expresión dinámica de una serie de procesos fisiopatológicos. Por lo tanto, es condición esencial evitar reduccionismos técnicos y resolver y entender que la aplicación de las maniobras no es simplemente un recurso nemotécnico para el estudiante. Tal repetición de maniobras, sin su revisión y juicio crítico, termina por desconocer el trasfondo biológico que se está viviendo.

Un segundo riesgo es pensar que los pacientes son todos similares entre sí y sus problemas pueden ser resueltos por una especie de axioma general único. Dicha idea debe ser apartada ya que en cada paciente existe una persona única $\mathrm{y}$, tal como es sabido, frente a una misma enfermedad existen diversas formas de reacción. De modo contrario se consideraría que todas las enfermedades son lo mismo en cada persona, como una especie de modelo universal, lo cual es falso, ya que según un adagio clínico clásico "más bien existen enfermos que enfermedades".

En tercer lugar, no podemos olvidar que la práctica de modelos de simulación es parte del proceso de la docencia médica. Educar implica enseñar y para ello se hace necesaria una condición esencial, cual es desear adquirir el conoci- miento para ponerlo en práctica de ahí en más. En este sentido, un párrafo de Besio y $\mathrm{Serani}^{3}$ explica mejor lo que decimos: "Lo esencialmente $h u$ mano de esa educación (profesional de la salud) tiene que ver con la adquisición de habilitaciones $o$ virtudes que tienen su asiento principal en la inteligencia y en la voluntad. Y antes en la voluntad que en la inteligencia. En efecto, toda cohorte de condiciones innatas, destrezas adquiridas y conocimientos 'acumulados' serían inoperantes si las personas que las adquirieron no las ponen al servicio del bien de los enfermos" Con ello queremos decir que lo que debe obtenerse de la educación con modelos de simulación, no es una mera suma de operaciones practicadas de modo automático, basadas en el cumplimiento estricto de protocolos y flujos de decisión o algoritmos memorizados. En una forma de educar de tal naturaleza advertimos el riesgo de un alejamiento de la relación médico-paciente, que debe rebosar de humanidad y reflexión, precisamente por la asimetría que se da en su estructura.

La utilización de modelos debe entenderse a la manera de una herramienta que facilite la enseñanza, pero en ningún caso puede sustituirla. El hecho más decisivo es que la técnica estructurada está pre-diseñada, con muchas soluciones y opera a la manera de entrenamiento, en una serie de repeticiones hasta lograr que la técnica se haga literalmente, "de memoria".

\section{Conclusión}

Pensamos que no hay un conflicto ético con la docencia por simulación en medicina, muy por el contrario. En la sociedad actual, se hace cada vez más necesario la práctica de técnicas y procedimientos, antes de ponerlos en práctica con pacientes reales. Existen razones para pensar e insistir en que hay motivos y fundamentos para sostener dos concepciones copulativas, esto es, que la simulación clínica es un bien en la medida que cumpla su compromiso intrínseco en cuanto fase preparatoria insustituible a la -igualmente insustituible- práctica clínica en pacientes reales en escenarios reales, y que ésta no será apropiada si lo anterior está ausente.

Una extrañeza moral sería pensar que la técnica es la forma global de enseñar medicina, dejando de lado el contacto con el enfermo de "carne y hueso", la mujer parturienta o el paciente que se encuentra en etapa terminal, ejemplos que ponen a prueba las mejores virtudes humanitarias del médico y hacen surgir la verdadera sabiduría en el arte de acoger y curar. 


\section{Bibliografía}

1.- PELÉS J, GOMAR C. El uso de las simulaciones en educación médica. Tesi 2010; 11: 147-69.

2.- PINAR G, PEKSOY S. Simulation-Based Learning in
Healthcare Ethics Education. Creative Education 2016; 7: 131-8.

3.- BESIO M, SERANI A. Sabiduría, naturaleza y enfermedad. Ediciones Pontificia Universidad Católica de Chile, Santiago, 2014, pág. 180.

Correspondencia a:

Dr. Alberto Rojas Osorio

Facultad de Medicina, Sede Viña del Mar,

Universidad Andrés Bello, Chile.

Email: arojas1040@yahoo.com 